\title{
The role of surfactants in Köhler theory reconsidered
}

\author{
R. Sorjamaa ${ }^{1}$, B. Svenningsson ${ }^{2}$, T. Raatikainen ${ }^{1}$, S. Henning ${ }^{2}$, M. Bilde ${ }^{2}$, and A. Laaksonen ${ }^{1}$ \\ ${ }^{1}$ Department of Applied Physics, University of Kuopio, P.O. Box 1627, 70211 Kuopio, Finland \\ ${ }^{2}$ Department of Chemistry, University of Copenhagen, Copenhagen, Denmark
}

Received: 26 March 2004 - Published in Atmos. Chem. Phys. Discuss.: 19 May 2004

Revised: 14 October 2004 - Accepted: 22 October 2004 - Published: 26 October 2004

\begin{abstract}
Atmospheric aerosol particles typically consist of inorganic salts and organic material. The inorganic compounds as well as their hygroscopic properties are well defined, but the effect of organic compounds on cloud droplet activation is still poorly characterized. The focus of the present study is the organic compounds that are surface active i.e. tend to concentrate on droplet surface and decrease the surface tension. Gibbsian surface thermodynamics was used to find out how partitioning between droplet surface and the bulk of the droplet affects the surface tension and the surfactant bulk concentration in droplets large enough to act as cloud condensation nuclei. Sodium dodecyl sulfate (SDS) was used together with sodium chloride to investigate the effect of surfactant partitioning on the Raoult effect (solute effect). While accounting for the surface to bulk partitioning is known to lead to lowered bulk surfactant concentration and thereby to increased surface tension compared to a case in which the partitioning is neglected, the present results show that the partitioning also alters the Raoult effect, and that the change is large enough to further increase the critical supersaturation and hence decrease cloud droplet activation. The fraction of surfactant partitioned to droplet surface increases with decreasing droplet size, which suggests that surfactants might enhance the activation of larger particles relatively more thus leading to less dense clouds. Cis-pinonic acidammonium sulfate aqueous solutions were studied in order to study the partitioning with compounds found in the atmosphere and to find out the combined effects of dissolution and partitioning behavior. The results show that the partitioning consideration presented in this paper alters the shape of the Köhler curve when compared to calculations in which the partitioning is neglected either completely or in the Raoult effect. In addition, critical supersaturation was measured for SDS particles with dry radii of $25-60 \mathrm{~nm}$ using a static paral-
\end{abstract}

Correspondence to: R. Sorjamaa

(riikka.sorjamaa@uku.fi) lel plate Cloud Condensation Nucleus Counter. The experimentally determined critical supersaturations agree very well with theoretical calculations taking the surface to bulk partitioning fully into account and are much higher than those calculated neglecting the partitioning.

\section{Introduction}

The indirect effect of atmospheric aerosols on climate change is still poorly characterized partly due to the lack of accurate composition data. The aerosol composition is found out to be diverse consisting of water, sulphates, nitrates, chloride salts, various types of organic compounds etc. Numerous studies have addressed the effects the inorganic compounds have on hygroscopic growth of atmospheric particles and cloud condensation nuclei $(\mathrm{CCN})$ activation, while the significance of organics has been recognized only recently (Charlson et al., 2001; Novakov and Penner, 1993).

In earlier studies the decrease in surface tension due to surface active organic compounds (SAOCs) has been considered, but the correct evaluation of surface and bulk concentrations (surfactant partitioning between the bulk and the surface of a droplet) has been neglected (Facchini et al., 1999; Shulman et al., 1996). Li et al. (1998) studied the effect of surfactant partitioning on surface tension with the mixture of sodium dodecyl sulphate (SDS) and sodium chloride $(\mathrm{NaCl})$ and found a reduction in Kelvin effect leading to an increase in critical supersaturation $\left(S_{c}\right)$. In this paper, we derive the Köhler theory accounting for surfactant effects rigorously from Gibbs' surface thermodynamics. We theoretically show that the partitioning of the surfactant between the bulk and the surface has to be considered not only when evaluating the Kelvin effect, but also in evaluating the Raoult effect. Our study also shows the effect of surfactants to depend on particle size. SDS together with sodium chloride is used to find out how the approach taken in this paper differs from the 


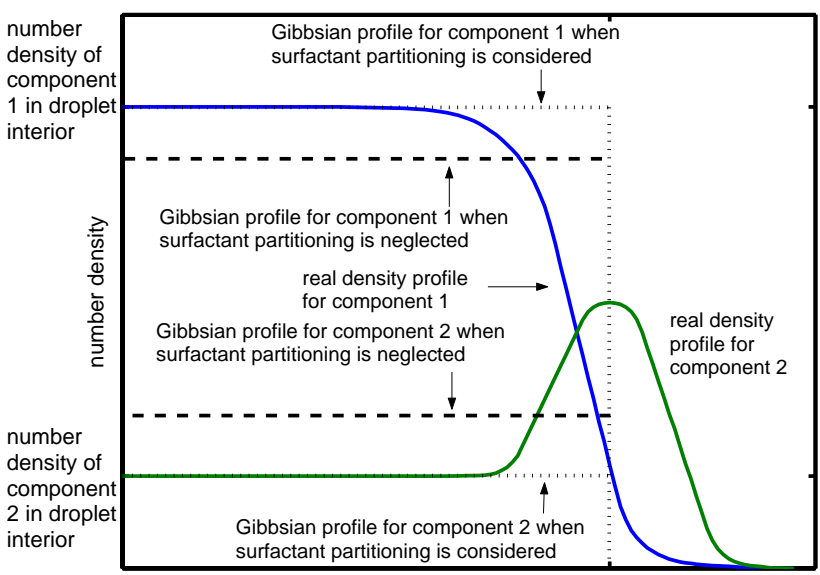

droplet radius

Fig. 1. Schematic presentation of density profiles in a two component case.

ones taken in earlier studies (Li et al., 1998; Facchini et al., 1999; Nenes et al., 2002; Abdul-Razzak and Ghan, 2004; Lohmann et al., 2004). We also study mixtures of cis-pinonic acid and ammonium sulphate to find out the effect that real atmospheric compounds may have on cloud droplet growth (Bilde and Svenningsson, 2004). Furthermore, we have also tested experimentally the results of different approaches in accounting for surface-active compounds and their surface to bulk partitioning during cloud droplet formation: an issue of high atmospheric relevance. The test was made for SDS particles, for which the surface to bulk effect on cloud drop activation should be large and relatively easy to detect experimentally.

\section{Theory}

The effects of organic compounds on droplet activation as well as the capability of organics to act as cloud condensation nuclei $(\mathrm{CCN})$ have been addressed in several studies (Cruz and Pandis, 1997; Hori et al., 2003; Kumar et al., 2003; Li et al., 1998; Shulman et al., 1996). In most of these studies the equilibrium growth has been described with Köhler theory

$$
S=\underbrace{\frac{2 \sigma M_{w}}{R T \rho_{w} r}}_{\text {Kelvin effect }}-\underbrace{\frac{3 n_{m} M_{w}}{4 \pi \rho_{w} r^{3}}}_{\text {Raoult effect }},
$$

where $S=$ supersaturation, $\sigma=$ surface tension, $M_{w}=$ molar weight of water, $R=$ ideal gas constant, $T=$ temperature, $\rho_{w}=$ mass density of water, and $r=$ droplet radius. Shulman et al. (1996) introduced a modified Köhler equation taking into account the solubility of a slightly soluble organic com- pound (oc) when evaluating the number of dissolved moles of solute in the droplet $\left(n_{m}\right)$

$n_{m}=\Phi_{o c} v_{o c} X_{d o c} n_{o c}+\Phi_{\text {salt }} v_{\text {salt }} n_{\text {salt }}$,

where $\Phi$ is molal osmotic coefficient, $v$ is the number of ions into which the compound dissociates, $X_{d o c}$ is the dissolved fraction of the organic compound, and $n_{o c}$ and $n_{\text {salt }}$ are the number of moles of organic compound and salt in the droplet, respectively.

\subsection{Surfactant partitioning in a two component system}

In reality the change from one bulk phase to another (e.g. from liquid to gas) does not take place in a stepwise fashion, but density profiles change smoothly over a region of a few molecular diameters. However, in Gibbs' surface thermodynamics, the change between the phases is taken to occur within an interfacial region of zero thickness called the Gibbs dividing surface. In the schematic Fig. 1, we show density profiles at the surface of a two-component droplet. The true total number of molecules of species $i$ in the droplet $\left(n_{i}^{t}\right)$ is obtained by integrating over the continuously varying density profile, while integration over the Gibbsian stepwise profile generally yields a different number, denoted by $n_{i}^{b}$. In the Gibbsian model, the so called surface excess number of molecules (which can be either positive or negative) corrects for this difference:

$n_{i}^{s}=n_{i}^{t}-n_{i}^{b}$.

Below, we will denote water by $i=1$ and the solute by $i=2$.

In most studies considering binary aqueous solutions with flat surface, the Gibbsian dividing surface is defined to exist at a location where the $n_{1}^{s}=0$ (called the equimolar surface). If the other component is an inorganic salt, the surface tension usually increases slightly as the salt concentration increases, which implies a slightly negative $n_{2}^{s}$ at the equimolar surface with respect to water. With a SAOC, the situation is different as it tends to concentrate on the surface, causing its density near the surface to become greater than in the droplet interior (Fig. 1). Thus $n_{2}^{s}$ has a large positive value at the equimolar surface with respect to water.

For flat surfaces, the location of the Gibbs dividing surface can be chosen freely because the value of the surface tension is independent of the choice. However, with curved surfaces the situation is different. For example, with spherical droplets, the value of the surface tension depends on the position of the dividing surface at which it is calculated. It can be shown (see e.g. Rowlinson and Widom, 1989) that if the surface tension is plotted as a function of distance from the droplet center, a minimum is found at a certain radius corresponding to the so called surface of tension. This minimum value is the effective surface tension of the droplet that appears in the well known Laplace equation $\Delta P=2 \sigma / r$ (the $r$ being the radius to the surface of tension and $\Delta P$ pressure difference between the center of the droplet and the vapor). 
In general, the value of the effective droplet surface tension depends on droplet size (Tolman, 1949). There are several papers addressing the problem of curvature dependence of fluids simpler than water (e.g. Schmelzer et al., 1996; Laaksonen and McGraw, 1996; Tsekov et al., 2000). The problem is already quite complicated with these simple fluids, and with water, estimates of the curvature dependence of surface tension can not be considered reliable (see e.g. Zakharov et al., 1997). With surfactant solutions, an extra degree of complication is added because the curvature dependence of surface tension is also dependent on the solution concentration (see e.g. Laaksonen and Napari, 2001). In practice, therefore, the curvature dependence is unknown and has to be neglected. However, when it comes to cloud droplets, this is no major setback as the curvature dependence becomes notable only for droplets smaller than about $10 \mathrm{~nm}$. In any case, it can be shown (Laaksonen et al., 1999) that there is a special case in which the curvature dependence of the surface tension vanishes altogether: if the surface of tension coincides with the special equimolar surface defined by

$$
\sum_{i=1}^{2} n_{i}^{s} v_{i}=0
$$

where $v_{i}$ is the molar volume of compound $i$, then the value of $\sigma$ equals the flat surface tension regardless of droplet size. We therefore adopt Eq. (4) in defining the location of the dividing surface. This choice differs from that of $\mathrm{Li}$ et al. (1998), who defined the dividing surface as the equimolar surface with respect to water (see above). For the droplet sizes considered in this paper, these two definitions do not lead to any recognizable differences, but the situation may be different with droplets smaller than $10 \mathrm{~nm}$.

To determine the surface and bulk concentrations for a droplet with known total numbers of molecules of both species, we apply the Gibbs adsorption equation:

$$
\sum_{i=1}^{2} n_{i}^{s} d \mu_{i}+A d \sigma=0,
$$

where $\mu_{i}=$ the chemical potential of compound $i, \sigma=$ the surface tension and $A=$ droplet surface area. The chemical potential is given as $\mu_{i}=k T \ln \gamma_{i} X_{i}$, with $\gamma_{i}$ the activity coefficient and $X_{i}$ the mole fraction of $i$. Because the interest of this study lies in the cloud droplet activation, assumption of dilute solution droplets is reasonable leading to activity coefficient of water becoming unity. The activity coefficient of the second compound (organic) may, on the other hand, be defined as follows

$\gamma_{2}=h X_{2}^{\nu_{2}-1}$

where $h$ is constant that depends on Henry's constant, saturation vapor pressure, and the degree of dissociation of the organic species, and $v_{2}$ denotes the number of ions the or- ganic molecule is dissociated to in dilute aqueous solution (Laaksonen et al., 1998). Thus,

$$
X_{2}=\frac{n_{2}{ }^{b}}{\sum_{i=1}^{2} v_{i} n_{i}^{b}} .
$$

From Eq. (4), it follows that the volume of the droplet is given by:

$$
V=\frac{4}{3} \pi r^{3}=\sum_{i=1}^{2} n_{i}^{t} v_{i}=\sum_{i=1}^{2} n_{i}^{b} v_{i} .
$$

From Eqs. (4)-(8) a simple iterative procedure gives the unknown surface excess for both compounds while the numbers of bulk moles are resolved from Eq. (3). As pointed out by Li et al. (1998), the surfactant partitioning affects the surface tension and thereby it also alters the critical radius and supersaturation. The Raoult effect describes the vapour pressure lowering of the solvent due to presence of solutes in the bulk of the liquid. What has not been noted before is that the surfactant partitioning therefore alters also the Raoult effect since the number of bulk moles differs from the total number of moles.

\subsection{Three component system}

The above equations can be applied also in a three component case just by extending the sums to go from one to three instead of one to two. Equation (6) is applicable for both the salt and the organic compound as long as they do not dissociate into similar ions. In the case of such mixed solution (for example $\mathrm{SDS}$ and $\mathrm{NaCl}$ both loosing a sodium ion), activity coefficients for both compounds have to be defined somewhat differently compared with Eq. (6). Thus, activity for ions $i$ and $j$ is (see e.g. Tester and Modell, 1997)

$a_{i j}=\gamma_{ \pm}^{v} X_{ \pm}^{v}$

$X_{ \pm}=\left[X_{i}^{v_{i}} X_{j}{ }^{v_{j}}\right]^{\frac{1}{v}}$

When Eq. (10) is applied to Eq. (9), the the activity coefficients for salt and organic compound become equal

$\gamma_{2}=\gamma_{3}=X_{2}+X_{3}$,

and thereby

$X_{2} d \mu_{2}=R T\left[\frac{X_{2}}{X_{2}+X_{3}}+1\right] d X_{2}$,

which is consistent with the equations of Li et al. (1998).

In a three component system with known total numbers of molecules in the droplet, there are six unknowns (the surface and bulk numbers of molecules for each component), however, the number of independent equations is only five, and therefore we must find an additional relation to be able to solve for the unknowns. Li et al. (1998) defined the dividing surface in the three component system in such a way that 
Table 1. Values for SDS and cis-pinonic acid properties required for the numerical model.

\begin{tabular}{lr}
\hline Property & Numerical value \\
\hline SDS density & $1.176 \mathrm{~g} \mathrm{~cm}^{-3}$ (Bittencourt, 1988) \\
Dissociation factor for SDS & 2 \\
Molal osmotic coefficient for SDS & 0.75 \\
Cis-pinonic acid density & $0.786 \mathrm{~g} \mathrm{~cm}^{-3}$ (Cruz and Pandis, 2000) \\
Dissociation factor for cis-pinonic acid & 1 \\
Molal osmotic coefficient for cis-pinonic acid & 1 \\
\hline
\end{tabular}

Table 2. Parameters for cis-pinonic acid solubility and surface tension parametrizations.

\begin{tabular}{lrr}
\hline Parameters & for solubility & for surface tension \\
\hline $\mathrm{a}$ & -0.15 & 0.003167 \\
$\mathrm{~b}$ & 1.46 & -0.041085 \\
$\mathrm{c}$ & 0.55 & 0.383714 \\
\hline
\end{tabular}

both $n_{1}^{s}$ and $n_{3}^{s}$ were set to zero, i.e. they assumed that the equimolar surfaces with respect to water and salt coincide. The surface deficiencies of salts in dilute binary solutions are usually quite small, and therefore we assume that the ratio of water and salt moles is practically constant as a function of droplet radius, giving us the following, sixth equation:

$\frac{X_{1}}{X_{3}}=\frac{n_{1}^{b}}{n_{3}^{b}}=\frac{n_{1}^{s}}{n_{3}^{s}}=\frac{n_{1}^{t}}{n_{3}^{t}}$.

This assumption leads to surface deficiencies of both water and salt when a surface excess of the organic compound exists. Since the approach taken by Li et al. (1998) only deals with one excess number, that of the SAOC, the molalities of salt and SAOC differ from those obtained with our model - yet the differences in the molalities are so small that they create only negligible differences in the Köhler curves.

Li et al. (1998) studied the effect of surfactant partitioning on cloud droplet activation with different mass fractions of sodium chloride $(\mathrm{NaCl})$ and sodium dodecyl sulphate (SDS) in the dry particle. We followed their study and made calculations for water-SDS and water-SDS- $\mathrm{NaCl}$ systems to find out what is the influence of surfactant partitioning on the Raoult effect and how it alters the critical radius and supersaturation.

\section{Numerical model}

As pointed out by Li et al. (1998) and Facchini et al. (2000), SDS is not a relevant atmospheric compound. Therefore the first simulations were done with $\mathrm{SDS}$ and $\mathrm{NaCl}$ simply to compare our theory to that presented in the paper by Li et al. (1998). SDS is also used to show how the effect of surfactants is dependent on particle size. Calculated results for pure SDS particles were also compared to measured results.

In the numerical model SDS is treated as an infinitely soluble compound and the calculations were made at $298.15 \mathrm{~K}$. Values for SDS properties required for numerical modeling are presented in Table 1. Surface tension parametrizations were the same as in Li et al. (1998). Mass weighted average density was assumed for dry particles, and as the activating droplets can be assumed dilute, the solution density was assumed to be that of water.

To gain an insight into the behavior of systems with more atmospheric relevance, we calculated Köhler curves for ammonium sulphate-cis-pinonic acid particles. The calculations were made at $273.15 \mathrm{~K}$ as in Shulman et al. (1996). Values for cis-pinonic acid properties required for numerical modeling are presented in Table 1. Cis-pinonic acid is a weak acid and therefore it was assumed not to dissociate while ammonium sulphate was taken to dissociate into three ions. As cis-pinonic acid is both a slightly soluble and a surface active compound, simple parametrizations were developed, for surface tension as well as for solubility $\left(M_{o c}^{\text {solub }}\right)$ using the data given in Shulman et al. (1996). Thus, $M_{o c}^{\text {solub }}=\frac{M_{o c}^{\text {solub }, w}}{1+a C_{\text {salt }}+b C_{\text {salt }}^{2}+c C_{\text {salt }}^{3}},\left(0<C_{\text {salt }}<3.0 \mathrm{moll}^{-1}\right)$, where $M_{o c}^{\text {solub,w }}$ is the solubility of cis-pinonic acid in pure water and $C_{\text {salt }}$ is the salt concentration. Parameters a, b and $\mathrm{c}$ are given in Table 2. The surface tension parametrization reads $\sigma_{\text {sol }}=\sigma_{w}+a C_{\text {salt }}+b\left(1+C_{\text {salt }}\right) C_{o c},\left(0<C_{\text {salt }}<2.0\right.$ moll ${ }^{-1}$ ), where $\sigma_{w}$ is the surface tension of pure water, and $C_{o c}$ and $C_{\text {salt }}$ are the concentrations of cis-pinonic acid and ammonium sulphate, respectively. Parameters $a, b$ and $c$ are given in Table 2 .

A question has been raised whether the surfactant partitioning is at all worth considering in atmospheric studies (Facchini et al., 2000). Our aim was to find out how this new surface to bulk partitioning consideration compares to other approaches and if the effect of partitioning on Köhler curve is worth noting for. Thus the Köhler curves were calculated with four different approaches: 
1. Without surfactant partitioning ( $X_{d o c}$ calculated using total number of surfactant moles, $n_{2}^{t}$ ) and with constant surface tension $\left(0.073 \mathrm{Nm}^{-1}\right)$

2. With surfactant partitioning $\left(X_{d o c}\right.$ calculated using bulk number of surfactant moles, $n_{2}^{b}$ ) affecting both surface tension and the Raoult effect

3. With surfactant partitioning affecting surface tension but not the Raoult effect (as in Li et al., 1998)

4. Without surfactant partitioning $\left(X_{d o c}\right.$ calculated using total number of surfactant moles, $\left.n_{2}^{t}\right)$, surfactant affecting surface tension $\left(\sigma=\sigma\left(X_{d o c}\right)\right)$.

\section{Experimental}

SDS $\left(\mathrm{C}_{12} \mathrm{H}_{25} \mathrm{OSO}_{3} \mathrm{Na}\right)$, purity $99 \%+$, was obtained from Sigma-Aldrich. Aqueous solutions of SDS were prepared using commercial water (Fluka, 17749) purified by reverse osmosis, ion exchange treatment and microfiltration.

Polydisperse aerosol was generated by atomizing aqueous solutions of SDS in an atomizer (TSI). After drying in diffusion dryers followed by dilution with dry clean air to relative humidities below $15 \%$ the particles were charged by exposing them to a $\mathrm{Kr}-85$ bipolar ion source. An almost monodisperse fraction of the aerosol was selected in a differential mobility analyzer (TSI, 3080), where the ratio of sheath to aerosol air in the DMA column was kept high $\left(10 \mathrm{lmin}^{-1} / 0.86-1.27 \mathrm{lmin}^{-1}\right)$. The monodisperse aerosol was divided between a condensation particle counter (TSI, 3010) and a static thermal-gradient diffusion cloud condensation nucleus counter, model CCNC-100B from the University of Wyoming, Department of Atmospheric Science (Snider and Brenquier, 2000; Delene and Deshler, 2000; Snider et al., 2003). The upper and lower surfaces of the chamber have moist surfaces (obtained by covering them with water-saturated blotter paper). Supersaturation inside the chamber is obtained by cooling the bottom of the chamber relative to the top.

The center axis of the chamber is illuminated by a laser diode $(670 \mathrm{~nm})$. Particles that are activated at the supersaturation present grow into droplets large enough to scatter light and are detected by measuring the forward scattered light intensity at an angle of 45 degrees to the laser diode light beam. The CCNC-100B supersaturation was calibrated using monodisperse $\mathrm{NaCl}$ and $\left(\mathrm{NH}_{4}\right)_{2} \mathrm{SO}_{4}$ particles as described in the paper by Bilde and Svenningsson (2004). The number concentration of particles entering the chamber were in the range 300-1000 and the temperature was in the range 297-301 K.

In order to account for possible non-spherical shape of the SDS particles (which could cause error in the experimentally determined dry particle size) they were studied with transmission electron microscope (TEM, JEM 1200 EX, manu-

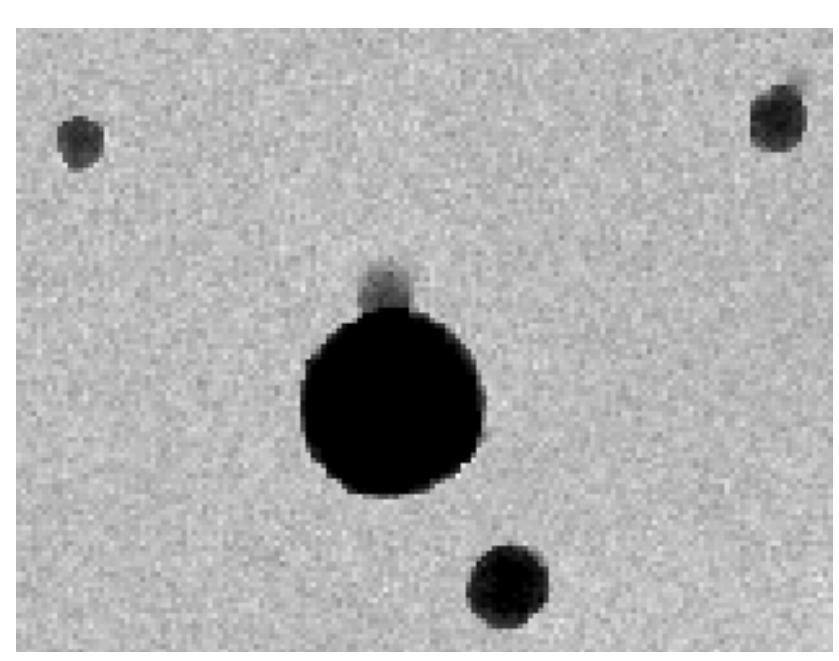

Fig. 2. TEM image of SDS particles. The diameter of the largest particle is approximately $150 \mathrm{~nm}$.

facturer JEOL Ltd., Japan). SDS particles were generated from aqueous solutions by a constant output atomizer with a dry air flow. The generated aerosol was then diluted with dry air thus leading to solid particles as the water evaporated from the droplets. The particles were then collected on a TEM-plate. The particles were spherical as can be seen from Fig. 2 and thus no shape correction for the comparison with experimental results was needed.

\section{Results}

\subsection{Two component system: SDS and water}

Even though SDS is not a relevant compound when it comes to atmospheric studies, it may still be used as a model compound to find out the effects that real atmospheric surfactants may have on droplet growth. The difference in surface thermodynamic formulation between our approach and the one taken by Li et al. (1998) would become significant if we were to study small droplets. However, this study concentrates on droplets large enough to act as $\mathrm{CCN}$ and thus the surfactant bulk concentrations obtained with these two approaches become approximately equal. Thereby the value of Kelvin effect obtained with our approach is equal to that given by the theory of Li et al. (1998). The real difference between our study and the study of Li et al. (1998) is that we account for the surface to bulk partitioning of the surfactant also when evaluating the Raoult effect.

For a particle with dry radius of $50 \mathrm{~nm}$, the critical radius is $0.262 \mu \mathrm{m}$ if the surfactant partitioning is included in calculation of both the surface tension and the Raoult effect, and $0.302 \mu \mathrm{m}$ if partitioning is taken to affect surface tension only (Table 3). The total amount of SDS was found to partition between droplet interior (bulk) and the surface in such a 
Table 3. Critical radii and supersaturations for Köhler curves calculated with different mass fractions of SDS and $\mathrm{NaCl}$ for a particle with dry radius of $50 \mathrm{~nm}$ and with $\mathrm{T}=298 \mathrm{~K}$. Approach 2 considers partitioning to affect on both surface tension and Raoult effect where as Approach 3 considers partitioning to affect only on surface tension (as in Li et al. (1998)).

\begin{tabular}{lllll}
\hline \multirow{2}{*}{$\begin{array}{l}\text { SDS mass } \\
\text { fraction }\end{array}$} & \multicolumn{2}{c}{ Approach 2 } & \multicolumn{2}{c}{ Approach 3 } \\
& $r_{c}(\mu \mathrm{m})$ & $S_{c}(\%)$ & $r_{c}(\mu \mathrm{m})$ & $S_{c}(\%)$ \\
\hline 0 & 0.688 & 0.103 & 0.688 & 0.103 \\
0.2 & 0.574 & 0.123 & 0.584 & 0.121 \\
0.5 & 0.423 & 0.167 & 0.452 & 0.157 \\
0.8 & 0.284 & 0.256 & 0.333 & 0.214 \\
1.0 & 0.262 & 0.304 & 0.302 & 0.247 \\
\hline
\end{tabular}

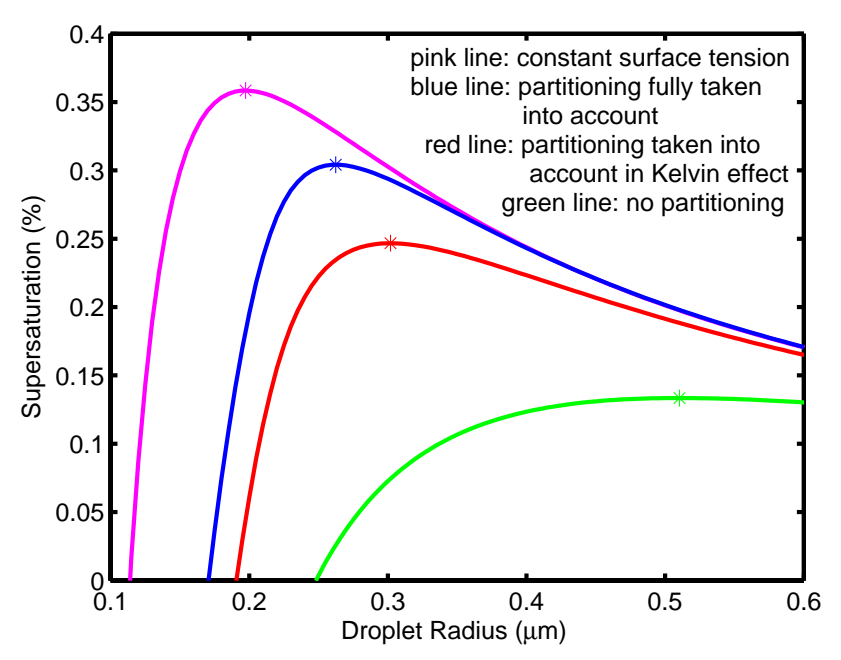

Fig. 3. Köhler curves for a SDS particle with dry radius of $50 \mathrm{~nm}$. The pink curve is for constant surface tension $\left(0.073 \mathrm{Nm}^{-1}\right)$ and for total number of moles in droplet bulk, the blue curve is calculated with surfactant partitioning affecting both Raoult effect and surface tension, the red curve accounts for partitioning affecting surface tension but not in Raoult effect and the green curve is for total amount of moles in droplet bulk. The asterisks represent the critical radius and critical supersaturation in each case.

way that the ratio $n_{2}^{s} / n_{2}^{b}=10.6$ at the critical radius, i.e. there is over ten times larger amount of SDS on droplet surface than in the bulk. Li et al. (1998) gave a value of 13.9 for this ratio. The difference between the values is due to different critical radii: the smaller the droplet the smaller surface area and thus there is a smaller amount of SDS molecules on droplet surface, and hence the value of $n_{2}^{s} / n_{2}^{b}$ is smaller when the partitioning is accounted for in the Raoult effect. Thus, the Raoult effect also becomes significantly smaller when the partitioning is accounted for, and in a two component case the effect on critical supersaturation is evident. If the surfactant partitioning is totally neglected, the peak of

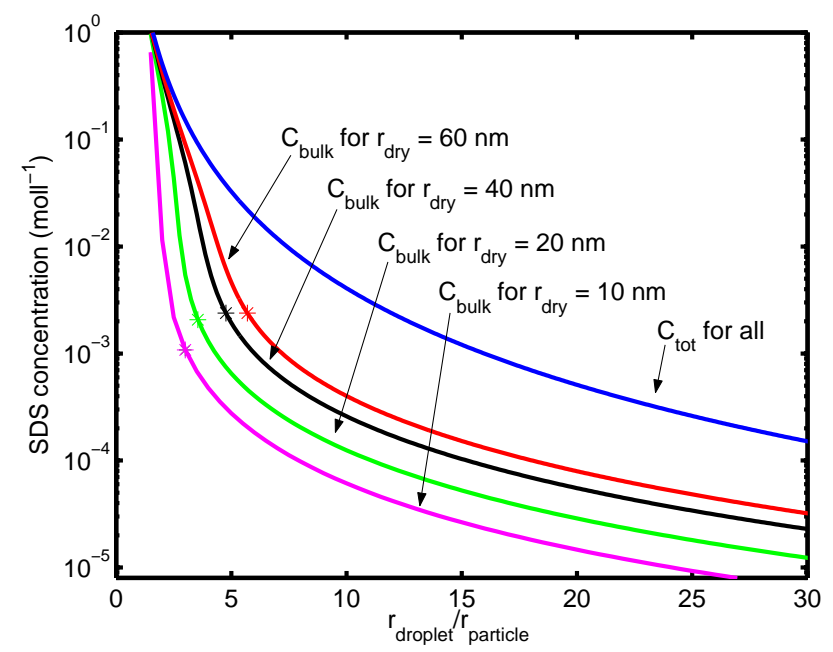

Fig. 4. SDS concentrations in droplets for four different dry particle radii $(10,20,40$, and $60 \mathrm{~nm})$. The $\mathrm{x}$-axis was chosen to present the ratio of droplet radius and dry particle radius to show that bulk concentration (red, black, green, and pink lines) depends on particle size where as the total concentration (blue line) does not. The asterisks show the surfactant concentration at critical particle size in each case.

the Köhler curve drops considerably and the activation becomes strongly overestimated as seen in Fig. 3. Actually, for a supposed atmospheric compound with thermodynamic properties similar to those of SDS, even the curve calculated with constant surface tension $\left(0.073 \mathrm{Nm}^{-1}\right)$ and no surfactant partitioning is closer to the correctly evaluated case than the case when the total amount of surfactant is considered to depress surface tension (Fig. 3).

The effect of surfactants becomes stronger for larger particles. This is due to the fact that the bigger the droplet the smaller the surface-to-volume ratio. Let us compare two droplets with different radii. If the ratio between droplet radius and particle dry radius is the same for both droplets, the overall concentration, i.e. the concentration calculated neglecting surfactant partitioning, and the bulk concentration are equal as well. When the surfactant partitioning is accounted for, the smaller droplet has a lower surfactant bulk concentration due to the relatively larger surface area as seen in Fig. 4. Thereby the effect of surfactant partitioning is greater with smaller particles resulting in increased surface tension and decreased Raoult effect when compared to larger particles. Figure 4 shows that when two droplets with same total surfactant concentration are being compared, the bulk concentration may differ significantly. The effect of particle size and partitioning on Köhler curves can be seen in Fig. 5. With a smaller particle and with correct partitioning evaluation (blue curve) the Köhler curve approaches the one calculated with surface tension of water (pink curve). With a larger particle the difference caused by the partitioning is not that drastic but still obvious. This brings about an interesting 


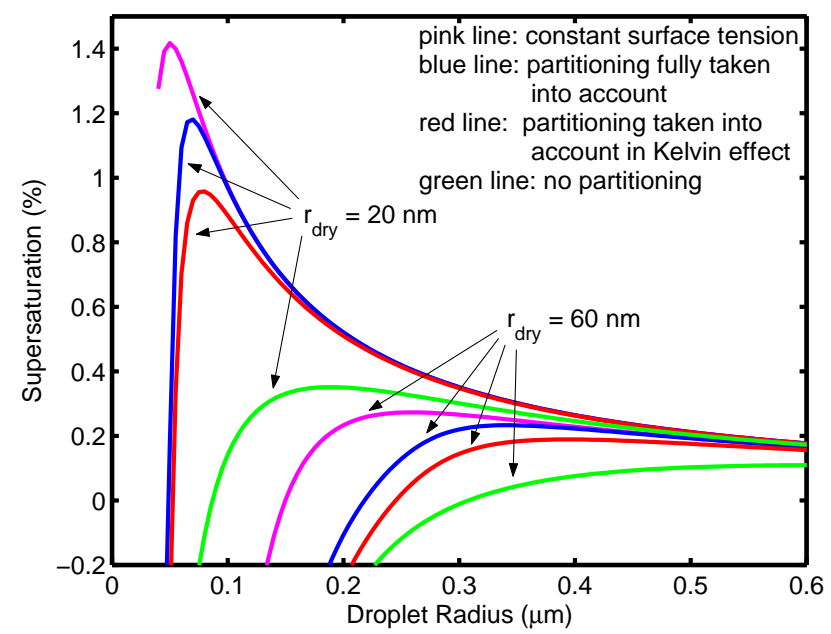

Fig. 5. Köhler curves for SDS particles with dry radius of $20 \mathrm{~nm}$ and $60 \mathrm{~nm}$. The pink curve is for constant surface tension $\left(0.073 \mathrm{Nm}^{-1}\right)$ and for total number of moles in droplet bulk, the blue curve is calculated with surfactant partitioning affecting both Raoult effect and surface tension, the red curve accounts for partitioning affecting surface tension but not in Raoult effect and the green curve is for total amount of moles in droplet bulk.

point concerning cloud model simulations: when the surfactant partitioning effects are properly accounted for, larger particles are more favored in cloud drop activation than when the partitioning is neglected, which may tend to decrease the cloud drop number concentrations.

5.2 Three component system: SDS, sodium chloride, and water

Since it is not realistic to expect atmospheric particles to be solely comprised of surfactants, the model was ran with different mass fractions of $\mathrm{NaCl}$ and $\mathrm{SDS}$ to see how the correct evaluation of surfactant partitioning effects compares to other approaches.

Köhler curves in Fig. 6 show that when the correct approach to surfactant partitioning is taken, the critical supersaturation may even exceed the one obtained with constant surface tension. Now the mass fraction of SDS in the dry particle is 0.5 and therefore there is less SDS to partition to the surface than in a two component case. On the other hand, $\mathrm{NaCl}$ present in the droplet tends to drive SDS to the surface and therefore the effect of partitioning on surface tension still remains considerable while the effect on the Raoult term becomes less significant ( $\mathrm{Li}$ et al., 1998). This is because there is a considerable amount of salt in the droplet compared to SDS and thereby the partitioning does not decrease the Raoult effect as clearly as in the two component case. Critical radii and supersaturations for a particle with $50 \mathrm{~nm}$ dry radius with different mass fractions of SDS are presented in Table 3.

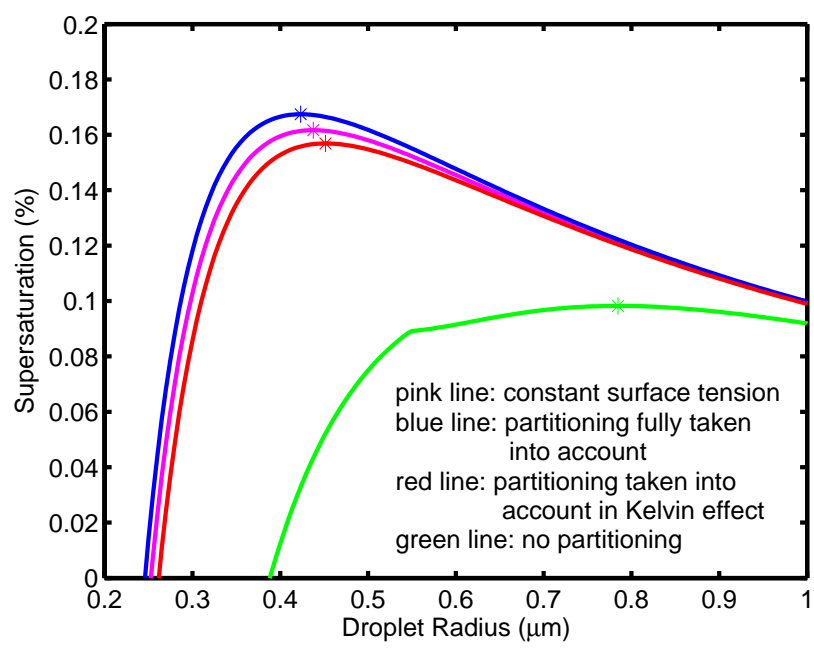

Fig. 6. Köhler curves calculated with same four partitioning considerations as in Fig. 3 for a particle with dry radius of $50 \mathrm{~nm}$ and mass fractions of SDS and $\mathrm{NaCl} 0.5$. The sharp corner in the lowest curve is due to discontinuity in the surface tension parametrizations.

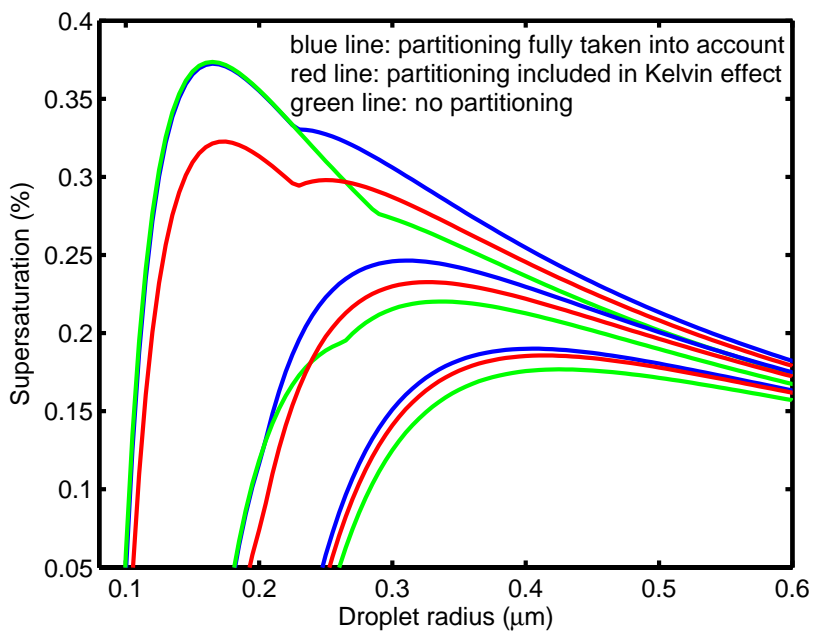

Fig. 7. Köhler curves calculated with three different approaches and with cis-pinonic acid mass fractions of 0.8 (three uppermost curves), 0.5 , and 0.2 (the three lowest curves). Blue curve is calculated with surfactant partitioning affecting both Raoult effect and surface tension, the red curve accounts for partitioning affecting surface tension but not in Raoult effect and the green curve is for total amount of moles in droplet bulk. Particle dry radius is $50 \mathrm{~nm}$.

\subsection{Three component system: Cis-pinonic acid, ammo-} nium sulphate, and water

Due to the assumption in Eq. (13), the salt molality is not affected by surfactant partitioning. As the solubility of cispinonic acid is dependent on salt concentration, also cispinonic acid bulk concentration remains independent of partitioning as long as there is a fraction of cis-pinonic acid not 


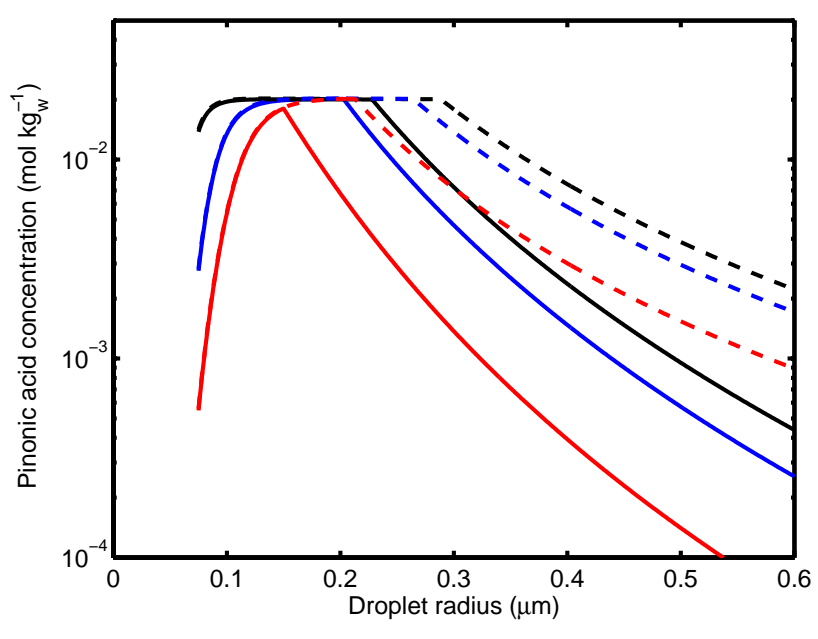

Fig. 8. Cis-pinonic acid bulk concentrations in a growing droplet. Particle dry radius is $50 \mathrm{~nm}$ and cis-pinonic acid mass fraction 0.2 (red line), 0.5 (blue line), and 0.8 (black line). Dashed lines represent the total concentrations, respectively.

dissolved. It has to be noted, however, that there is a surface excess also when cis-pinonic acid is dissolving, and therefore surface partitioning increases the total amount of dissolved cis-pinonic acid for a droplet of a given size. Because of this, the transition radius $r_{T}$ (at which the cis-pinonic acid is completely dissolved) is reached earlier when the partitioning is accounted for (Fig. 7). Before complete dissolution the cis-pinonic acid bulk concentration is determined by its solubility, and because the bulk concentration defines both the surface tension and Raoult effects, the Köhler curves obtained using our approach and with the approach of Shulman et al. (1996) are identical up to the radius $r_{T}$ of our approach.

The partitioning starts to cause difference in the surfactant bulk concentrations and the Köhler curves part from each other right after complete dissolution. Now the situation is similar to the case with SDS and $\mathrm{NaCl}$ : as the droplet grows its surface area increases, causing an increasing number of cis-pinonic acid molecules to partition to the surface. Figure 8 shows the cis-pinonic acid bulk concentrations of the droplets in Fig. 7. Black, blue, and red lines represent the cis-pinonic acid bulk concentrations with different dry particle mass fractions, and the dashed lines represent the total concentrations, respectively. The greater the salt mass fraction in the particle the smaller the cis-pinonic bulk concentration as long as salt concentration is greater than $0.1 \mathrm{M}$. When ammonium sulphate concentration reaches $0.1 M$ the cis-pinonic acid solubility, i.e. the bulk concentration, becomes constant (horizontal part of the curves in Fig. 8). For particles with cis-pinonic acid mass fraction of 0.5 (blue line) and 0.8 (black line) there is still an undissolved fraction left when $0.1 M$ salt concentration is reached. Those two lines part as soon as complete dissolution occurs for a particle with

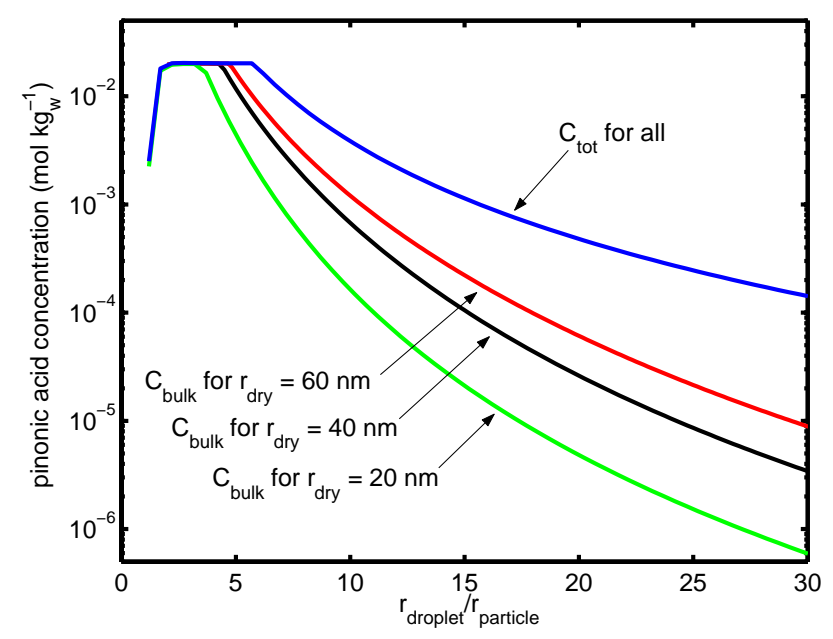

Fig. 9. Cis-pinonic acid concentrations in droplets for three different dry particle radii $(20,40$, and $60 \mathrm{~nm})$. Bulk concentration (red, black, and green) depends on particle size where as the total concentration (blue line) does not. Cis-pinonic mass fraction is 0.8 .

smaller cis-pinonic acid mass fraction. When the cis-pinonic acid mass fraction is 0.2 , the complete dissolution takes place before the $0.1 M$ salt concentration is reached (red line). This solubility behavior can also be seen in Fig. 9 together with the effect of particle size. The particle size has the same effect in a three component system as it has in a droplet consisting solely of surfactant and water as in Fig. 4.

In the case of a slightly soluble compound, ignoring the partitioning in the evaluation of the Raoult effect leads to a clear discrepancy in the whole partitioning scheme. The Kelvin effect is correctly estimated, as partitioning is considered to affect surface tension. But when the partitioning is neglected in the Raoult effect, $n_{m}$ includes also surface moles and is thereby overestimated. That is why the Köhler curve is depressed until the transition radius is reached (Fig. 7). After complete dissolution the situation is similar to the case with SDS and $\mathrm{NaCl}$, the difference between the red and green Köhler curve in Fig. 7 is caused by the correct surface tension evaluation and the difference between the red and blue curve is due to disparity in the Raoult effect. The differences between the approaches become naturally more pronounced as cis-pinonic acid mass fraction increases.

Hegg et al. (2001) have experimentally investigated the $\mathrm{CCN}$ activity of pinonic acid. They found a decrease in cloud drop number concentration when compared to inorganic compounds. The magnitude of this decrease might be diminished in a polydisperse aerosol population by the accelerated dissolution, which is caused by surfactant partitioning. This conclusion can be drawn from the Fig. 7 as the particle with mass fraction of 0.5 and dry radius of $50 \mathrm{~nm}$ shows smaller critical radius when the partitioning is accounted for. 


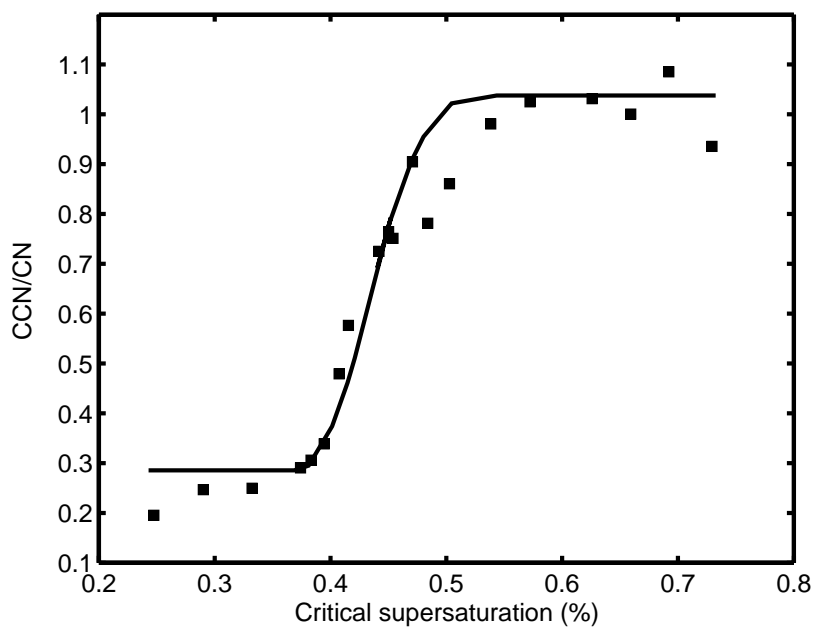

Fig. 10. Activated fraction as a function of supersaturation for particles with dry radius of $40 \mathrm{~nm}$. The solid line represents the fitting made in order to find the critical supersaturation.

\subsection{Comparison with experimental results}

The number fraction of SDS particles that were activated to cloud droplets was measured as a function of supersaturation in the CCN counter for particles with dry radii of $25,30,35$, $40,45,50,55$, and $60 \mathrm{~nm}$, respectively. In all cases, a welldefined activation was observed. As an example, data for particles with dry radius of $40 \mathrm{~nm}$ are presented in Fig. 10. The effect of particles passing the DMA as doubly charged, thus being larger than the singly charged, can be seen as a background level in the activated fraction of about 0.3 for supersaturations below $0.4 \%$. The critical supersaturation for $40 \mathrm{~nm}$ particles is determined as the supersaturation for which $50 \%$ of the singly charged particles are activated. An S-shaped curve, the slope of which is based on the width of the particle size distribution defined in the DMA, is fitted to the data (Fig. 10).

Critical supersaturations as a function of particle dry radius are presented in Fig. 11. Error bars denote 95\% confidence intervals based on calibration with ammonium sulphate and sodium chloride. Experimental results obtained by Rood and Williams (2001) are also shown. The agreement between the two experimental data sets is good. Comparison with theoretical results is made with the four different approaches described in Sect. 3 and presented e.g. in Figs. 3-5. For SDS, a very surface active and water-soluble compound, the experimental results agree well with calculations taking the surface to bulk partitioning into account in both Raoult's law and the Kelvin effect (blue line in Fig. 11).

The experimental results presented here support the theoretical treatment and confirm that the surface area is of importance also for water soluble surfactants in systems where the surface area to volume ratio is as large as in

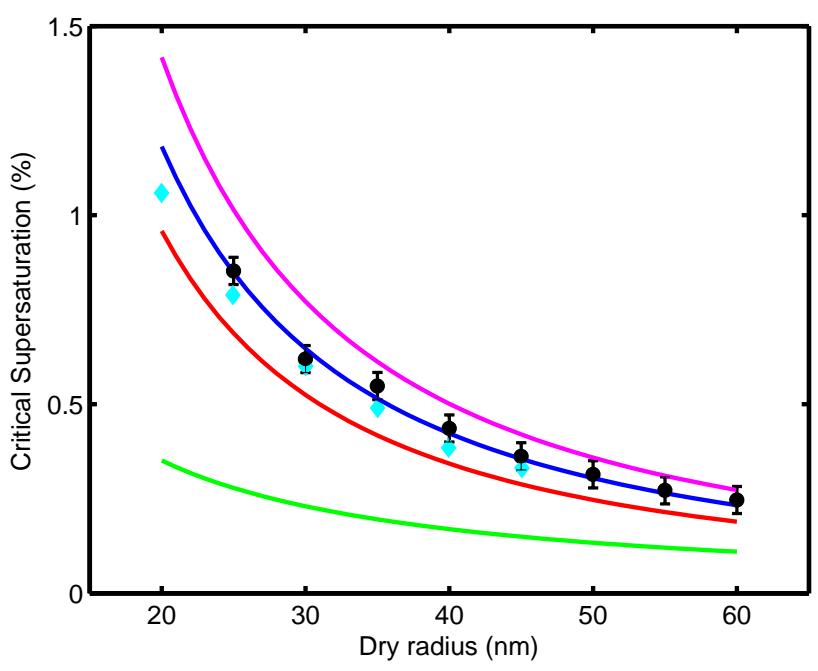

Fig. 11. Experimental critical supersaturations for eight different particle dry radii (circles) with error bars representing 95\% confidence intervals. The data from Rood and Williams (2001) is plotted with turquoise diamonds for comparison. The lines represent the theoretical calculations. The pink line is for constant surface tension $\left(0.073 \mathrm{Nm}^{-1}\right)$ and for total number of moles in droplet bulk, the blue line is calculated with surfactant partitioning affecting both Raoult effect and surface tension, the red line accounts for partitioning affecting surface tension but not in Raoult effect, and the green line is for total amount of moles in droplet bulk.

sub-micrometer activating droplets. The effect is very pronounced for SDS, increasing the critical supersaturation with a factor of 3 for a $25 \mathrm{~nm}$ radius particle and with a factor of 2 for a $60 \mathrm{~nm}$ radius particle compared to the case where surface tension data are used, but the partitioning is not taken into account. Neglecting the partitioning would also lead to the erroneous conclusion that organic compounds like SDS are more efficient as $\mathrm{CCN}$ than ammonium sulfate. However, a correct theoretical treatment and experiments show much higher critical supersaturations for SDS than for inorganic salts of atmospheric relevance. The same mechanism should be relevant for water-soluble surfactants in the atmosphere. Support for this can be found in the work by Decesari et al. (2003). They showed that SDS, fulvic acid, cloud and fog water samples as well as dissolved aerosol material exhibit the same type of response in dynamical surface tension measurements, in contrast to insoluble surfactants. However, presently it is not possible to quantify the effect of surface partitioning of surfactants for unknown and complex mixtures like atmospheric aerosol particles. The theoretical treatment described above can only be used for well-known, relatively simple mixtures. 


\section{Conclusions}

As pointed out by Shulman et al. (1996), a slightly soluble compound affects the shape of the Köhler curve by gradual dissolution and by depressing the critical supersaturation due to lower surface tension. Our study shows that this is the case also when the surfactant partitioning is accounted for.

However, the partitioning causes the slightly soluble surfactant to dissolve completely at an earlier stage of droplet growth. The present study also revealed that the critical supersaturation may be underestimated if surfactant partitioning is neglected. This is because not only the surface tension is affected by the partitioning but also the Raoult effect as the partitioning causes the number of solute molecules in droplet bulk to decrease. A water soluble compound, i.e. SDS, showed that in some cases with large enough organic mass fraction the critical supersaturation could actually reach even a higher value when compared to an approach with no partitioning consideration or surface tension lowering.

The results also show that the smaller the particle the greater the effect of surface to bulk partitioning, and thus the effect of surfactants becomes more significant with larger droplets. This indicates that the surfactants could actually enhance the growth of the larger droplets and lead to less dense clouds. Thus, the presence of a soluble and/or slightly soluble surfactant in a cloud droplet may alter droplet activation, but the magnitude of this alteration depends entirely on particle chemical composition and size, and on environmental variables.

The theoretical and experimental results obtained in this study clearly show the importance of surfactant partitioning evaluation. From the theoretical point of view it is obvious that the partitioning needs to be considered in evaluation of both the Kelvin and the Raoult effects. This has been shown with a strong surfactant (SDS) and compound of atmospheric relevance (cis-pinonic acid) as well as with mixtures of inorganic salt and surfactant. The two sets of experimental data for critical supersaturations of pure SDS particles are both in very good agreement with the model results taking the surface to bulk partitioning into account in the Raoult term as well as in the Kelvin term. The data also confirms that the surface area is of importance also for water soluble surfactants in systems where the surface area to volume ratio is as large as in sub-micrometer activating droplets. We expect that the same mechanism is relevant for water-soluble surfactants in the atmosphere and this gives rise to a series of new questions: how important is the effect in the atmosphere? How do we describe complicated mixtures theoretically? To address these questions the next step will be to study atmospherically relevant mixtures.
Acknowledgements. The study has been funded by the Academy of Finland (project number 201052 and through the Center of Excellence program), Danish Natural Science Research Council, and Swiss Funding Agency. We also acknowledge the support from the Nordic Center of Excellence, Research unit on BiosphereAtmosphere-Cloud-Climate-Interactions (BACCI). We thank Pasi Miettinen for the help with particle generator.

Edited by: B. Kärcher

\section{References}

Abdul-Razzak, H. and Ghan, S. J.: Parameterization of the influence of organic surfactants on aerosol activation, J. Geoph. Res., 109, D03205 doi:10.1029/2003JD004043, 2004.

Bilde, M. and Svenningsson, B.: CCN activation of slightly soluble organics: the importance of small amounts of inorganic salt and particle phase, Tellus B, 56, 128-134, 2004.

Bittencourt, D. R. S.: X-Ray powder diffraction data for sodium octyl sulfate, sodium decyl sulfate and sodium dodecyl sulfate Powder Diffr., 3, 244, 1988.

Charlson, R. J., Seinfeld, J. H., Nenes, A., Kulmala, M., Laaksonen, A., and Facchini, M. C.: Reshaping the theory of cloud formation, Science, 292, 2025-2026, 2001.

Cruz, C. N. and Pandis, S. P.: A study of the ability of pure secondary organic aerosol to act as cloud condensation nuclei, Atm. Environ./, 31/, 2205-2214, 1997.

Cruz, C. N. and Pandis, S. P.: deliquescence and hygroscopic growth of mixed inorganic-organic atmospheric aerosol, Environ. Sci. Technol./, 34/,4313-4319, 2000.

Decesari, S., Facchini, M. C., Mircea, M., Cavalli, F., and Fuzzi, S.: Solubility properties of surfactants in atmospheric aerosol and cloud/fog water samples, submitted to J. Geophys. Res., 108, 10.1029/2003JD003566, 2003.

Delene, J. D. and Deshler,T.: Calibration of a photometric cloud condensation nucleus counter designed for deployment on a balloon package, J. Atmos. Ocean Tech., 17, 459-467, 2000.

Facchini, M. C., Mircea, M., Fuzzi, S., and Charlson, R. J.: Cloud albedo enhancement by surface-active organic solutes in growing droplets, Nature, 401, 257-259, 1999.

Facchini, M. C., Mircea, M., and Fuzzi, S.: Comments on "influence of soluble surfactant properties on the activation of aerosol particles containing inorganic solute", J. Atmos. Sci., 58, 14651467, 2000.

Hegg, D. A., Gao, S., Hoppel, W., Frick, G., Caffrey, P., Leaitch, W. R., Shantz, N., Ambrusko, J., and Albrechcinski, T.: Laboratory studies of the efficiency of selected organic aerosols as CCN, Atmos. Res., 58, 155-166, 2001.

Hori, M., Ohta, S., Murao, N., and Yamagata, S.: Activation capability of water soluble organic substances as ccn, J. Aerosol Sci., 34, 419-448, 2003.

Katz, J. L. and Mirabel, P.: Calculation of supersaturation profiles in thermal diffusion cloud chamber, J. Atmos. Sci., 32, 646-652, 1975.

Kumar, P. P., Broekhuizen, K., and Abbat, J. P. D.: Organic acids as cloud condensation nuclei: Laboratory studies of highly soluble and insoluble species, Atmos. Chem. Phys., 3, 509-520, 2003. 
Laaksonen, A. and McGraw, R.: Thermodynamics, gas-liquid nucleation, and size-dependent surface tension, Europhys. Lett., 35, 367-372, 1996.

Laaksonen, A., Korhonen, P., Kulmala, M., and Charlson, R. J.: Modification of the Köhler equation to include soluble trace gases and slightly soluble substances, J. Atmos. Sci., 55, 853862, 1998.

Laaksonen, A., McGraw, R., and Vehkamäki, H.: Liquid-drop formalism and free-energy surfaces in binary homogeneous nucleation theory, J. Chem. Phys., 111, 2019-2027, 1999.

Laaksonen, A. and Napari, I.: Breakdown of capillarity approximation in binary nucleation: a density functional study, J. Phys. Chem. B, 105, 11 678-11 682, 2001.

Li, Z., Williams, A. L., and Rood, M. J.: Influence of soluble surfactant properties on the activation of aerosol particles containing inorganic solute, J. Atmos. Sci., 55, 1859-1866, 1998.

Lohmann, U., Broekhuizen, K., Leaitch, R., Shantz, N., and Abbatt, J.: How efficient is cloud droplet formation of organic aerosols?, Geophys. Res. Lett., 31, L05108, doi:10.1029/2003GL018999, 2004.

Nenes, A., Charlson, R. J., Facchini, M. C., Kulmala, M., Laaksonen, A., and Seinfeld, J. H.: Can chemical effects on cloud droplet number rival the first indirect effect?, Geophys. Res. Lett., 29, 1848-1851, 2002.

Novakov, T. and Penner, J.: Large contribution of organic aerosols to cloud-condensation-nuclei concentrations, Nature, 365, 823826, 1993.

Rood, M. J. and Williams, A. L.: Reply, J. Atmos. Sci., 58, 14681473, 2001.
Rowlinson, J. S. and Widom, B.: Molecular theory of capillarity, Clarendon Press, Oxford, 1989.

Schmelzer, J. W. P., Gutzow, I., and Schmelzer Jr., J.: Curvaturedependent surface tension and nucleation theory, J. Colloid Interface Sci., 178, 2, 657-665 (9), 1996.

Shulman, M. L., Jacobson, M. C., Charlson, R. J., Synovec, R. E., and Young, T. E.: Dissolution behavior and surface tension effects of organic compounds in nucleating cloud drops, Geophys. Res. Lett., 23, 277-280, 1996.

Snider, J. R. and Brenguier, J. L.: Cloud condensation nuclei and cloud droplet measurements during ACE-2, Tellus, 52B, 828842, 2000.

Snider, J. R., Guibert, S., Brenquier, J.-L., and Putaud, J.-P.: Aerosol activation in marine stratocumulus clouds: $2 \mathrm{~K} /$ "ohler and parcel theory closure studies, J. Geophys. Res., 108 (D15), 8629, doi:10.1029/2002JD002692, 2003

Tester, J. W. and Modell, M.: Thermodynamics and its applications, Prentice-Hall, 1997.

Tolman, R. C.: Consideration of the gibbs theory of surface tension, J. Chem. Phys., 17, 118, 333-337, 1949.

Tsekov, R., Stöckelhuber, K. W., and Toshev, B. V.: Disjoining pressure and surface tension of a small drop, Langmuir, 16, 35023505, 2000.

Zakharov, V. V., Brodskaya, E. N., and Laaksonen, A.: Surface tension of water droplets: a molecular study of model and size dependencies, J. Chem. Phys. 107, 24, 10 675-10 683, 1997. 